\title{
Maximising the educational and research value of the undergraduate dissertation in psychology
}

*Ann-Marie Creaven ${ }^{a}$ https://orcid.org/0000-0002-2467-307X

${ }^{a}$ Department of Psychology, University of Limerick, Limerick, Ireland.

Katherine S. Button ${ }^{\mathrm{b}}$ https://orcid.org/0000-0003-4332-8789

${ }^{b}$ Department of Psychology, University of Bath, U.K.

Heather Cleland Woods ${ }^{c}$ orcid.org/0000-0003-3382-5925

c School of Psychology and Neuroscience, University of Glasgow, Scotland

Emily Nordmann ${ }^{\mathrm{c}}$ https://orcid.org/0000-0002-0806-1081

d School of Psychology and Neuroscience, University of Glasgow, Scotland

*Corresponding author.

The authors report no conflicts of interest.

Version 2: this preprint was reworked following initial reviews. The literature review has been expanded and the reliance on personal supervisory experience has been reduced. The models for supervision have been combined to better reflect their underlying commonalities, a table and a figure have been added for clarity, and we have provided five clear recommendations to help support supervisors.

Please note that this pre-print version is yet to undergo peer-review.

Acknowledgements: We are very grateful to Professor Neil Coulson (University of Nottingham) for his helpful contribution to and feedback on an earlier draft of this manuscript and to Dr Peter Branney (Bradford University) for feedback also.

We are also grateful to the National Forum for the Enhancement of Teaching and Learning in Higher Education who supported a seminar on this topic as part of the 2020/2021 seminar series (recording here: https://www.youtube.com/watch?v=7iBVt2ZqPCo) 


\begin{abstract}
The undergraduate research dissertation in psychology is the capstone demonstration of research skills including project planning and design, considering and resolving ethical issues, and the analysis and dissemination of findings. The dissertation represents an opportunity for learning as well as an opportunity to contribute to the research literature in the student's chosen area; however, few articles have considered both of these dimensions in detail. This article provides a roadmap for undergraduate thesis supervision, for early-career supervisors and supervisors aiming to better align their supervision and research activities and/or engage their students in open research practices via the dissertation.

Specifically, we review prior literature on undergraduate psychology research supervision and identify a number of dimensions that vary in existing approaches. Drawing on our own supervision experiences, we describe further approaches and discuss how these can support student learning as well as benefit research. We conclude by identifying five key recommendations for undergraduate supervision in psychology that support student learning and research efficiency and integrity.
\end{abstract}


The undergraduate (UG) research dissertation/thesis in psychology requires the student to carry out an empirical piece of research over the course of a single academic year. Students must individually demonstrate a range of research skills including project planning and design, considering and resolving ethical issues, and the analysis and dissemination of findings (British Psychological Society [BPS], 2019; Psychological Society of Ireland [PSI] 2019). Although the dissertation is an important learning process for individual UG students, it may also contribute to the research literature in the student's chosen area, in the form of grey literature, or a peer-reviewed publication. Therefore, completing a dissertation has individual pedagogical benefits for the student, and potentially wider benefits for the research literature. The purpose of this paper is to discuss the dissertation as both a pedagogical exercise and a research endeavour and consider how to best support students' learning while also enhancing the potential benefits for research. In doing so we hope to make the "private realm" (Wiggins et al., 2016, p. 11) of dissertation supervision explicit. We conclude by emphasizing key considerations for undergraduate supervision in psychology.

There is an extensive literature already on the value of undergraduate involvement in research (Perlman \& McCann, 2005), models of research involvement aside from the dissertation (e.g., Lloyd et al., 2019), considerations specific to qualitative dissertations (e.g., Freeman et al., 2020), effective mentorship (e.g., Boysen et al., 2020), and postgraduate research (e.g., Williams, 2019). To be clear, our focus is not on those topics but on modes of undergraduate dissertation supervision that support both efficiency in research and highquality learning for students. This is timely given advances in open science practices intended to improve the efficiency, reliability, and accessibility of research outputs.

Traditionally, the dissertation has been conducted in the context of a dyadic relationship between supervisor and student and involves the collection of new data. The dissertation context may magnify the impact of some questionable research practices (QRPs, 
John et al., 2012), as the sheer numbers of student projects with limited time and resources can result in small sample sizes and low power for quantitative projects, offering fertile ground for false positive findings. Undergraduate psychology students do report engaging in QRPs, particularly in relation to analysis and reporting (Krishna \& Peter, 2018). Further, dissertation supervisors have been identified as key in shaping students' attitudes towards QRPs (Krishna \& Peter, 2018), and a 2016 survey of over 100 researchers (Baker, 2016) identified better teaching and training in research methods as one of the key levers for bringing about cultural change and improving research integrity. Therefore, the undergraduate dissertation is a particularly valuable opportunity to train the next generation in rigorous research practices and research integrity.

Reviewing recent literature indicates that approaches to supervision vary across several domains. These are:

(1) the extent to which the dissertation research question and study design is student-led versus supervisor-led (see Knight \& Botting, 2016, for a discussion in relation to allied health dissertations)

(2) the extent to which the dissertation is conducted as a primarily individual (with no meeting besides one-to-one meetings with the supervisor) or group (with only group meetings) endeavour (see Button et al., 2019; Dautel, 2020; Mickley-Steinmetz \& Reid, 2019)

(3) the presence of a single or multiple supervisors, formally or informally (e.g., as part of a cluster dissertation model involving 2 formal supervisors; Freeman et al., 2020; or a consortium-model involving doctoral /post-doctoral researchers in addition to multiple supervisors; Button et al., 2019) 
(4) the extent to which the dissertation builds explicitly on prior research experience (e.g., as a continuation of research conducted on placement; see Healey et al., 2013), or is a standalone endeavour

(5) collection of primary data versus the use of existing data sets (e.g., Schultz et al., 2005, for a broad discussion).

(6) the extent to which dissertation findings are required to be formally or informally disseminated (e.g., via class or conference presentation, or as a journal submission)

In Table 1 we summarise models of undergraduate thesis supervision documented for psychology, beyond the traditional dyadic model. This is not an exhaustive summary of approaches but rather provides an overview of alternatives to the dyadic model. Each of the models described involve some form of team approach. All models differ slightly in their setup and hierarchies but the potential pedagogical benefits of all include enhanced peer support and the development of teamwork skills. Other advantages include greater efficiency and potentially greater engagement in the project for staff. However, as team approaches become increasingly popular, they will require formal evaluation (Boysen et al., 2020). 
Table 1. Summary of models of undergraduate thesis supervision in psychology.

\begin{tabular}{|c|c|}
\hline Reference & Overview \\
\hline $\begin{array}{l}\text { Button et al. (2019) - } \\
\text { Consortium Model }\end{array}$ & $\begin{array}{l}\text { Groups of students across multiple universities collaborate on a common research protocol under the supervision of their } \\
\text { local academic supervisor, and a PhD student or post-doctoral researcher. Students design their dissertations to align with } \\
\text { this larger multi-site study and meet with their supervisor as a group. The common protocol addresses an overarching } \\
\text { research question and students test secondary hypotheses for their dissertations. }\end{array}$ \\
\hline $\begin{array}{l}\text { Mickley-Steinmetz \& Reid } \\
\text { (2019) - Apprentice-Based } \\
\text { Senior Thesis }\end{array}$ & $\begin{array}{l}\text { Groups of 3-6 students collaborate on common research protocols under a single academic supervisor. This model is } \\
\text { similar to the consortium model run on a smaller scale (i.e., in a single institution rather than across multiple } \\
\text { universities). }\end{array}$ \\
\hline $\begin{array}{l}\text { Dautel (2020) - Collective } \\
\text { Academic Supervision }\end{array}$ & $\begin{array}{l}\text { A group of students undertake independent, but related, research projects, under the supervision of a common supervisor. } \\
\text { Students meet only on a group basis in this instance. }\end{array}$ \\
\hline $\begin{array}{l}\text { Detweiler-Bedell \& } \\
\text { Detweiler-Bedell (2019) - } \\
\text { Laddered Model }\end{array}$ & $\begin{array}{l}\text { Students are organised into three-person laddered teams, with an experienced student (team leader) mentoring a mid- } \\
\text { level student alongside a student new to the research group. Though not specific to dissertation supervision, the duration } \\
\text { of involvement in the laddered team over multiple years can facilitate dissertation supervision for the student acting as } \\
\text { team leader. }\end{array}$ \\
\hline $\begin{array}{l}\text { Freeman et al. (2020) - } \\
\text { "Cluster" projects }\end{array}$ & $\begin{array}{l}\text { In the context of qualitative supervision, two members of staff are paired together to supervise a group of students (in } \\
\text { addition to one-to-one supervision), for projects that operate around one or more of the following: (a) a single topic, (b) a } \\
\text { specific research question, (c) a specific method or set of methods, (d) a specific approach to data analysis. }\end{array}$ \\
\hline $\begin{array}{l}\text { Wagge et al. (2019) - } \\
\text { Collaborative Replication } \\
\text { Education Project (CREP) }\end{array}$ & Students participate in high-quality direct replications selected and overseen by the CREP team. \\
\hline $\begin{array}{l}\text { Moshontz et al. (2018) - } \\
\text { Psychological Science } \\
\text { Accelerator (PSA) }\end{array}$ & $\begin{array}{l}\text { The PSA is a distributed network of laboratories designed to enable and support crowdsourced research projects. Unlike } \\
\text { CREP, it is not explicitly focused on either replications or on students; however, it is a mechanism within which } \\
\text { undergraduate theses may be conducted. }\end{array}$ \\
\hline
\end{tabular}


Several benefits for research are evident in these team approaches. For qualitative dissertations, a team approach facilitates peer learning and potentially mentorship from multiple supervisors (Freeman et al., 2020), as well as opportunities for a junior mentor to develop their supervision skills. For quantitative projects, team approaches can additionally increase statistical power and the likelihood of generating a publishable journal article. The consortium model (Button et al., 2019) minimizes QRPs by mandating pre-registration of the primary hypothesis and enables students to contribute to a peer-reviewed publication led by an early-career researcher (ECR) with oversight over the multi-site data collection. This ECR also gains valuable experience in a principal investigator (PI) role. In the laddered model (Detweiler-Bedell \& Detweiler-Bedell, 2019), where post-doctoral level ECRs are uncommon, students gain experience as team assistants in the first instance. By the time they undertake their own dissertations as team leaders, they are well-placed to contribute to co-authoring a journal article with their mentor (while team associates and assistants are acknowledged, where appropriate). Building on these approaches, we present further alternatives to the traditional model that may be realistic to implement with limited resources and discuss how these might (a) enhance the learning inherent in the dissertation process and (b) contribute to enhancing the quality of research that students generate as part of their dissertations. As illustrated in Figure 1, the models fall into two broad categories (a) the use of secondary/existing data and (b) the use of a team science approach to collect primary data. All models described align with the BPS and PSI Standards/Guidelines for Accreditation (BPS, 2019; PSI, 2019). 
Figure 1. Overview of study designs with supporting resources to enhance learning and research efficiency in the undergraduate dissertation process
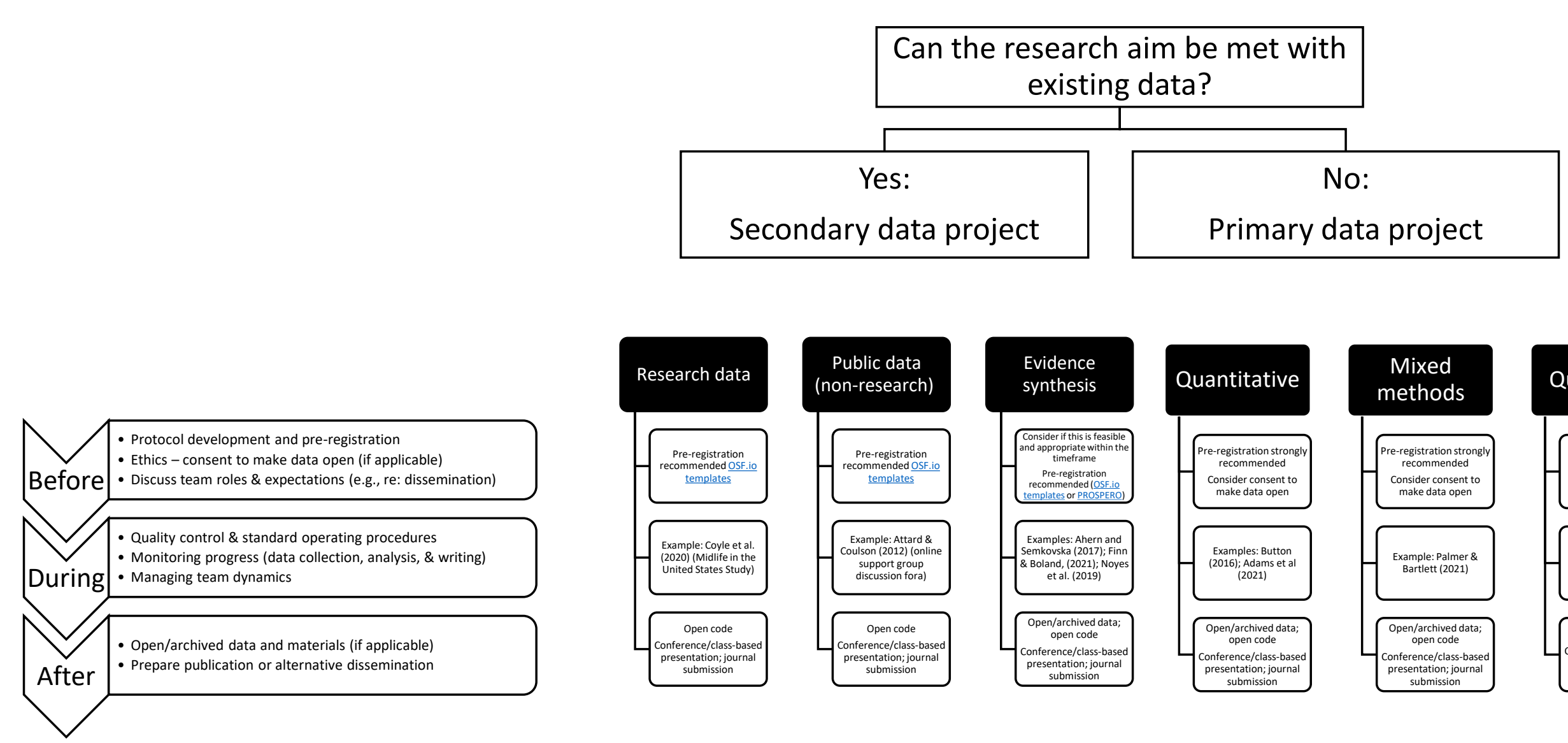

Examples are undergraduate or masters projects (either published
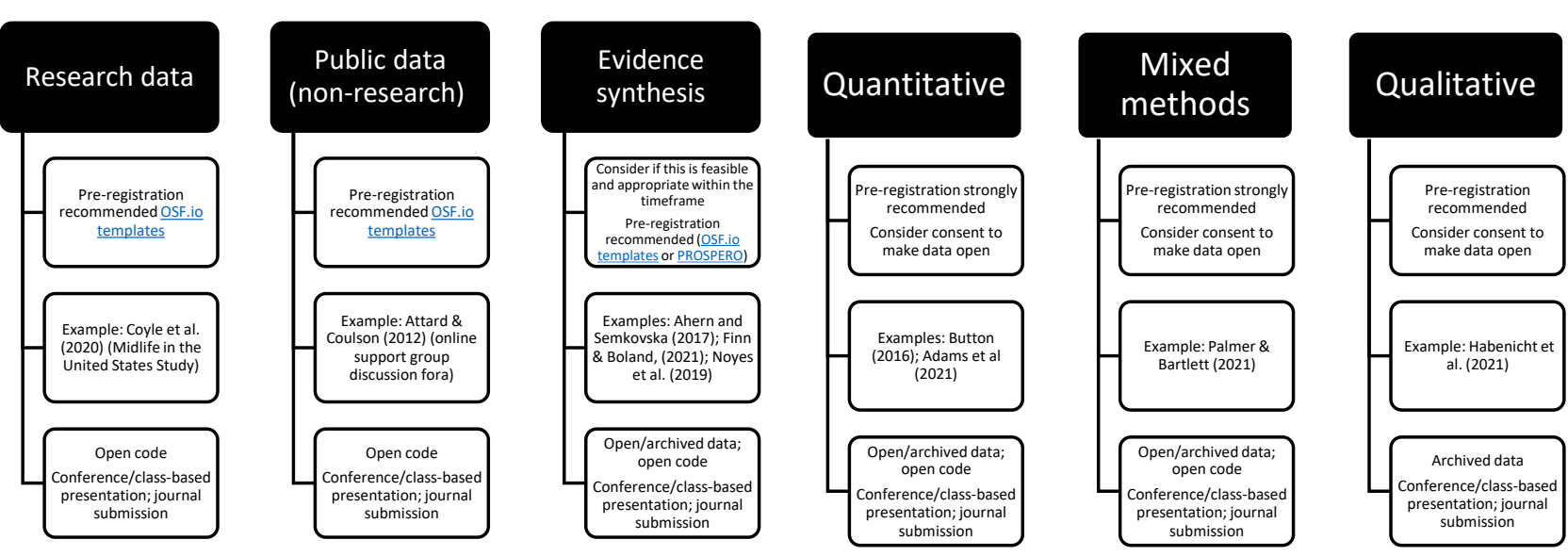


\section{Secondary data analysis}

Many research data sets (quantitative and qualitative) are publicly available and easily accessible online; others require a brief application to obtain access (see here for a list of datasets: https://osf.io/th8ew/). Usually, data collection methods are well-documented, a large sample size is available, and sampling is more representative of the general population than would be achieved with convenience or snowball sampling. Prior published studies using such data can give students a sense of what a strong empirical contribution could look like. Many of these advantages also apply to datasets already collected by the supervisor or student. Additionally, publicly available data not originally intended for research purposes, for example, Twitter or discussion forums, can be a rich source of data that does not add to the volume of research waste.

In this model, students will not gain first-hand experience of participant recruitment or data collection. Depending on individual School/Department requirements, students may not gain experience of developing a formal research ethics application, so considering how to develop competency in ethics is necessary, for example, by creating ethics forms to relate directly to secondary data and/or internet-mediated research. Ethics is particularly important for data not originally collected for research purposes. Researchers relying on data from online forums, for example, need to consider if individual forum members should be contacted for permission to analyse their online discussions. If appropriate, researchers need to consider if it is feasible and if it could alter the nature of the online discussions. There may be alternatives such as seeking permission from a forum administrator, or they may be grounds for researchers to choose not to seek consent. These considerations are complex (see e.g., Ahmed et al., 2017; Buchanan, 2017 for further discussion) and there is no clear right or wrong answer. Indeed, for large-scale Twitter analyses that scrape data from a particular hashtag, informed consent is practically impossible to obtain. Instead, it is important to 
consider a formal application for access via Twitter's Academic Research application, and to ensure users are granted anonymity in the write-up and/or the publication of data and analysis code. For example, Attard and Coulson (2012) used data in the public domain and thus did not seek consent. To preserve anonymity, they omitted not only participants' names/ pseudonyms but also the names of the online support groups themselves, and only short segments of the original posts were quoted to reduce their traceability through search engines. In addition to this example, useful guidance on relevant ethical issues is available from the BPS (2017) (see also Granger et al., (2021), Sugiura et al., (2017) and Williams et al., (2017)).

It is important to consider that large-scale studies from which secondary datasets may be drawn often make do with brief versions of psychometric measures, or constructs similar, but not identical to, the construct of interest. Constructs might be described using unfamiliar terminology (e.g., items measuring "social connectedness" or "social integration" might be described as items relating to "social exclusion"). Students will need more time to familiarize themselves with the data format, files, and coding conventions (e.g., for missing data) than they would for data and scales they constructed themselves. Additionally, secondary data may involve aggregated data (e.g., total scores may be reported rather than individual scale items). Access to more fine-grained data may involve a more stringent application process and new versions (containing additional variables, cases, or correction of errors) may be released from time to time. Students should be aware of and supported to deal with these issues.

The most straightforward projects involve a single, easily accessible, cross-sectional dataset. More complex designs may involve matching several data sets, issues of attrition between different elements of a study (e.g., questionnaire and telephone interview) and different naming conventions for variables collected at different time-points. Students' skills 
in data wrangling may require support at the start, particularly where they have encountered only unrealistically "clean" data and the skills required to obtain data from sources like Twitter are likely to only be available to those students who are enrolled in psychology programmes that have incorporated data skills and programming into their curriculums (e.g., PsyTeachR, n.d.).

Although publications using the data are not a substitute for technical documentation, students may find them useful. Replication studies may also be feasible (e.g., Coyle et al., 2020). The advantages of replication for learning and guidance on choosing what to replicate for teaching purposes are discussed in-depth elsewhere (see Janz, 2016; Wagge et al., 2019). Unlike studies involving shared primary data collection, there is no explicit advantage in using a team approach. However, the same secondary dataset could be used for a group of students, who can duplicate each other's code and analyses. Similarly, a team approach might lend itself well to discussions of the ethical issues relating to the particular data set, and/or to data management and open data. Quantitative secondary data analyses can still be preregistered to support students to focus on their specific research questions, with templates available to facilitate this (e.g., Weston et al., 2021). Finally, although qualitative secondary analysis is less commonly done, there is increasing commentary on methodological and ethical issues (e.g., Ruggiano \& Perry, 2017; Tate \& Happ, 2018) to support this.

\section{Evidence synthesis}

Conducting an evidence synthesis allows students to engage deeply with the literature and develop their methodological and appraisal skills. For dissertation projects adhering to British and Irish standards, our interpretation of current accreditation guidelines is that data analysis must be conducted; therefore, a systematic review without a meta-analysis (or equivalent) is unlikely to be acceptable in Britain and Ireland (e.g., BPS, 2020), while U.S. 
guidelines are less prescriptive. University libraries often offer training in systematic review techniques and there are many published exemplars available. An evidence synthesis can be undertaken even if others have previously been conducted addressing the same question. For example, Ahern and Semkovska (2017) addressed some limitations of an earlier metaanalysis (Lee et al., 2012) of cognitive functioning in the first episode of major depressive disorder. For qualitative evidence synthesis (QES, see Noyes et al., 2019) a student will ideally have prior experience with the methodology they are synthesizing (e.g., students undertaking thematic syntheses, will have experience in thematic analysis), which is unlikely at UG level. However, working with data in existing papers where themes are already summarized, is arguably more accessible than working with raw qualitative data, making qualitative evidence synthesis viable option for some students under some specific circumstances. Similar to the use of preregistration templates, students undertaking an evidence synthesis can use the PRISMA (Page et al., 2021) guidelines to help scaffold and guide their project, as well as encouraging transparent reporting.

For this model, it is important to ensure students appreciate the distinction between the narrative and selective literature review that forms part of an assignment or research project, and the substantial workload involved in undertaking a systematic review prior to even conducting a meta-analysis. In contrast to narrative reviews, at least some steps of a systematic reviews should be conducted as part of a team (Jahan et al., 2016). The availability of team members may determine whether a systematic review is a feasible option for the dissertation. Additionally, the supervisor must consider the accreditation requirement for meta-analysis. Given the typical timeframe for UG dissertations, it may be helpful to consider if the research question and process can be constrained to reduce the burden associated with the searching and screening phases of the process. For example, it may be possible to update a previous review, or conduct a review of research during the last five 
years, or from the date a key research recommendation was made. Finally, supervisors and students should also plan for insufficient or inadequate reporting of data for meta-analysis. Although contacting the study authors is commonly done to access data, there is no guarantee authors will be responsive. If at least some data are available, conducting a meta-analysis while acknowledging the limitations of available data may demonstrate students' computational skills. However, given the considerable time required to conduct evidence syntheses well, and the accreditation requirement for data analysis, this option is often suboptimal for UG students.

\section{A. Team science (quantitative)}

Teaming up across institutions in the consortium model (Button et al., 2019) can lead to very large datasets and more generalizable results. However, many of the benefits can be achieved by groups of students working together within an individual department. The BPS/PSI accreditation guidance endorses group projects as long as the student can individually demonstrate each of the skills involved in conducting the empirical project, whilst the APA guidelines include refining project-management skills and enhancing teamwork capacity as core goals. Depending on the institution's interpretation of the guidance, this could be as simple as each student writing up their dissertations separately (based on identical research questions and a common dataset) or ensuring that each student has a different research question.

For quantitative studies, similar to the consortium model, group lab or survey projects can be supported by having an overarching primary hypothesis or project aim that is pre-registered and forms the primary focus of any paper written up for publication. Students then build in a series of secondary questions and hypotheses to become the focus of their individual dissertations. A key concern of this approach is the tension between pedagogy and 
research. The consortium lab-based example incorporates multiple outcomes and/or moderator variables for pedagogical reasons (i.e., to facilitate individual student research questions), and this complexity increases with the number of students in the team. However, the integrity of the overarching project (on which all students will be co-authors) is retained by pre-registering the primary aims. Thus, any resulting publication will be confirmatory for the primary aims, with the students' dissertation aims treated as secondary. Pre-registering (even informally) individual student hypotheses ensures that the dissertation projects retain their individuality, both in terms of academic integrity, and the perception of the process from the students' point of view. It also minimizes the temptation to make use of measures other than those that were pre-registered after the data have been collected.

\section{B. Team science: Mixed methods}

Mixed methods designs involve the collection and analysis of both quantitative and qualitative data and as such lend themselves well to a team dissertation project. Creswell and Clark (2018) identify three core mixed method designs: convergent (qualitative and quantitative data collection and analysis is conducted largely in parallel), explanatory (qualitative data is collected subsequent to quantitative analysis to help explain the results), and exploratory (qualitative data is collected prior to quantitative collection and analysis to help guide the quantitative design and hypotheses. Mixed methods provide both breadth and depth to the question under investigation (Johnson et al., 2007) and in the case of exploratory mixed methods, can support the formation of evidence-based hypotheses for NHST (Erzberger \& Prein, 1997). Specific to the dissertation process, mixed methods projects have several advantages. First, students can support each other closely without concerns over originality and individual skill development. The shared topic means that they can engage in peer support such as sharing papers and discussing theoretical models and the interpretation 
of their data, however, the divergence in methods and subsequent write-up ensures that the dissertation project retains its individuality. Additionally, students can support each other with participant recruitment and reviewing and proofreading study materials. Team mixed method designs also allow (or indeed may require) multiple supervisors to be involved on the project who have different methodological expertise. Students still benefit from an individual supervisor, but group meetings and reviews help promote a team science approach.

One important consideration with mixed method dissertations involves the timeline and the choice of core design. By definition, explanatory and exploratory designs require one student to "go first", which may make convergent designs more appealing. Supervisors should be clear upfront about the nature of the project and be prepared to support those students with different timelines than they may have expected; for example, completing drafts of the introduction and methods in advance of any data collection. It is crucial that contingency plans (e.g., switching to a convergent design) are developed for if the primary study does not take place in the expected timeline, so that for example, issues impacting student A do not disadvantage student B. Additionally, the quantitative component remains susceptible to the limitations of individual quantitative projects (e.g., small sample sizes). There are of course higher-level concerns with mixed method designs regarding how to meaningfully integrate studies that have different epistemological positions into a single paper (Clark, 2019), although this is not specific to mixed method research as a dissertation model.

\section{C. Team Science: Qualitative Research}

Finally, teaming up for fully qualitative research is effective when students are interested in distinct but related questions with the same participant group (e.g., exploring experiences of 
(a) social support and (b) self-management for students with Type 1 diabetes). It is most straightforward when students also plan to use the same analytical approach (e.g., thematic analysis), as the same "thickness" of data is sought during interviews, and there are no differences in transcription requirements (e.g., text only transcription is acceptable for both research questions).

In addition to sharing participant recruitment, students can review each other's materials such as interview or focus group questions before finalising a common schedule. Students can divide the conduct of interviews/focus groups and transcribe those they do not conduct, to gain familiarity with the data. In contrast to exploratory or explanatory mixed methods designs, students work to the same timeline for data collection.

The key concern with pairing up for qualitative studies involves assuring the quality and originality of the two individual research studies within the common research process. Students must clearly articulate their own specific research questions prior to combining question schedules. Otherwise, they may end up conducting several interviews or focus groups around the broad topic while ending up with very little material pertinent to their specific research question. Student A must also be sufficiently familiar with Student B's research question, to probe and follow up on participant responses relating to that question (and vice versa). Students can decide a priori to analyse the interviews in their entirety, or alternatively, that only about half of each interview will be relevant for each dissertation. Even with training, there is potential for a high level of variability in interview skills and establishment of rapport, and any section on reflexivity will be increasingly complex with increasing number of interviewers/researchers.

\section{Team Science Approaches: Conclusion}


Regardless of the methodological or epistemological stance of the dissertation, students can be supervised as a team and benefit from peer support and learning, and potentially greater student engagement and a higher rate of on-time completion (Akister et al., 2009). They can co-write study protocols or interview schedules, and pool their recruitment, data collection and transcription efforts and get real experience of working in a research team. However, students may feel they have less scope for input into the study design. As noted by Dautel (2020), students may also miss out on the benefits of one-to-one meetings with their supervisor. Therefore, a hybrid model involving a team approach complemented by some one-to-one interaction could be preferable, if possible.

Besides this, the social and emotional aspects of learning and of the dissertation are also important to consider. Students can gain a sense of pride having completed a solo dissertation that they may not feel to the same degree working on a team-based project; this could be addressed by highlighting individual achievements within the team as well as team-work overall. Students progressing from a team-based dissertation to a solo project for masters or $\mathrm{PhD}$ research may be vulnerable to "impostor syndrome" or find the transition disconcerting. Therefore, scaffolding transitions from team to solo projects (as you would for transitions from solo to team projects) may be necessary.

\section{Student-led research designs}

Finally, whilst some dissertations are closely aligned to existing supervisory projects, it is also the case that many supervisors will allow their students to design their own study, in recognition of the capstone nature of the dissertation in a student's academic journey. These student-led projects are rarely originally intended to be submitted for publication, with the view that this is a small price to pay for giving the student the pedagogical experience that can come with increased independence and the freedom to make mistakes. However, student- 
led research designs may add an extra stage at which publication bias can arise, when such a project turns out to be a "diamond in the rough" - a student who is fortunate enough to generate results that turn out to be exciting and novel. These projects should have the same likelihood of being submitted for publication as those student-led projects that have strong designs but end in null results, or whose dissertations may require more extensive rewriting before publication, yet this is unlikely to be the case. The risk of false-positives is further inflated by student-led designs that may not have had the same level of supervisory oversight as a researcher-led project (in light of the pedagogical benefits of making mistakes) and where sample sizes may have been determined by the student's social reach. The independence of student-led research designs is not to be discouraged; however, it is important to protect against the added risk such projects may involve.

\section{Discussion}

Based on the supervision experiences described above, we argue that adopting five key recommendations may help increase the quality of the research generated as part of the UG dissertation, without compromising, and potentially increasing, the quality of student training and learning. These recommendations are:

(1) Consider efficient use of or generation of data (and open data)

(2) Consider a team approach

(3) Pre-register the research (formally or informally)

(4) Model and scaffold transparency in data analysis

(5) Identify opportunities for dissemination

(1) Consider efficient use of or generation of data (and open data) 
First, consider whether data should be collected at all, to address the research question. Data collection involves practical skills development; however, if these skills can be acquired outside of the dissertation, the use of existing data might be especially appealing. Evidence synthesis is also an option, though we do not strongly recommend it for the reasons outlined above. If data are collected, consider making the data "open" in line with FAIR data principles (Wilkinson et al., 2016). Although there is debate about the value of open data initiatives (e.g., Kitchin, 2013), and it is highly challenging to truly anonymise data (e.g., Rocher et al., 2019), making a conscious decision to make data open or not is an important element of student training given increasing emphasis on open data and data management in general. These considerations are relevant to qualitative data (see Branney et al., 2019).

\section{(2) Consider a team approach}

Second, consider a team approach. This can facilitate practical data collection skills while making efficient use of the data collected. Supervisors contemplating this may be concerned about research integrity and independent working. However, clarifying to students which activities can be shared (e.g., circulating relevant research papers), and which should not be done collaboratively (writing one's individual results sections), can be helpful in supporting the retention of individual research integrity within a larger team project. Other strategies include balancing team meetings with some individual meetings focused on the student's own specific research question and intellectual development, and bringing the team together for particular types of activities (e.g., pilot testing in the lab), before dispersing the team for other activities.

In our experiences, students have valued both the informal peer support that comes from working in this way, and the tangible advantages of shared data collection; these benefits have been highlighted by others, also (e.g., Dautel, 2020). Moreover, if social 
loafing is a concern, this may be mitigated a priori by clear discussions and agreement on roles and responsibilities, and/or by requiring individuals to collect a certain proportion of data to access the larger shared data set. Students value opportunities for one-on-one discussions with their supervisor, so a combination of team and individual meetings is possibly most beneficial (Dautel, 2020). There may also be instances where students have very legitimate concerns about working together and in these cases, an individual project (perhaps involving secondary data) may be more appropriate.

\section{(3) Pre-register research (formally or informally)}

Full pre-registration with detailed analytic specificity is not appropriate for all research designs and analytic approaches (and indeed there is ongoing debate as to its efficacy and purpose for any research, see e.g., Nosek et al., (2019) vs. Szollosi et al. (2019)). However, incorporating the development of a study protocol, lighter-touch pre-registration of hypotheses for confirmatory work, or the explicit registration that the work is exploratory/intended solely as a learning experience, is entirely feasible and can help promote transparent ways of working and protect against the increased risk of publication bias in the wider literature (Pownall, 2020). From a learning perspective, working through a detailed plan prior to data collection will inevitably lead to clarity of thinking and better research questions and higher quality dissertations. Indeed, van't Veer and Giner-Sorolla (2016) note that the focus on validating theory and method over results is likely to benefit researchers at an earlier stage of their career, specifically students and post-doctoral researchers. Further, while reproducibility and replicability are relevant to quantitative, rather than qualitative, approaches, the importance of transparency has long been recognised in qualitative constructs such as reflexivity, the process of a continual internal dialogue and critical self-evaluation of a researcher's positionality as well as active acknowledgement that 
this position may affect the research process and outcome (Berger, 2015). Therefore, preregistration is also a tool to support transparency in qualitative research.

\section{(4) Model and scaffold transparency in data analysis}

In addition, analytic transparency and reproducibility should be encouraged for quantitative work. Whilst only a small number of psychology departments around the world currently teach statistics using programming languages such as R (Wills, n.d) that by their nature promote reproducibility, students using point-and-click software, such as SPSS, can still be asked to provide syntax files to ensure the results reported in the dissertation can be reproduced. Similarly, qualitative dissertation students can generate reflexivity or positionality statements as part of their theses.

\section{(5) Identify opportunities for dissemination}

Finally, regarding dissemination, to be clear, we are not advocating that all student dissertations are published. The dissertation is first and foremost a learning exercise, and the pressure to publish can result in feelings of inadequacy on the part of students when findings are not deemed publishable (Roberts \& Seaman, 2019). However, there is an ethical case for ensuring that the data collected from research participants contributes to the wider research base; if it is not intended to constitute publishable research, this should be made clear to participants in advance, so that they can make an informed decision to participate. Aside from ethical considerations, the incentives associated with publication extend to undergraduate research, and there is increasing interest in publishing with undergraduates (Giuliano et al., 2019). Publication is also an important part of the research process. By collaborating on a journal submission, students develop scientific writing skills and gain hands-on experience of disseminating research. Therefore, we recommend that dissemination 
is incorporated into the dissertation process, if not formally as a journal submission, then locally as a presentation.

\section{Conclusion}

To conclude, the UG dissertation is both a pedagogical exercise and a traditional, albeit small-scale, research project. This paper combining our supervision experiences aims to make some of this "private realm" (Wiggins et al., 2016) explicit and provide recommendations on how to support learning outcomes as well as the overall quality of undergraduate research. Given the increasing emphasis on open science practices, and increasing popularity of team approaches, formal evaluation of the impact on student learning is an important next step in this area. 


\section{References}

Adams, R. C., Button, K., Hickey, L., Morrison, S., Smith, A., Bolus, W., Coombs, E., Randolph, S., Hunt, R., Kim, D., Chambers, C. D., \& Lawrence, N. S. (2021). Foodrelated inhibitory control training reduces food liking but not snacking frequency or weight in a large healthy adult sample. Appetite, 167, [105601]. https://doi.org/10.1016/j.appet.2021.105601

Ahern, E., \& Semkovska, M. (2017). Cognitive functioning in the first-episode of major depressive disorder: A systematic review and meta-analysis. Neuropsychology, 31(1), 52-72. http://dx.doi.org/10.1037/neu0000319

Ahmed, W., Bath, P. A., \& Demartini, G. (2017). Using Twitter as a data source: An overview of ethical, legal, and methodological challenges. The Ethics of Online Research. https://doi.org/10.1108/S2398-601820180000002004

Akister, J., Williams, I., \& Maynard, A. (2009). Using group supervision for undergraduate dissertations: a preliminary enquiry into the student experience. Practice and Evidence of the Scholarship of Teaching and Learning in Higher Education, 4(2), 77-94.

Attard, A., \& Coulson, N.S. (2012). A thematic analysis of patient communication in Parkinson's disease online support group discussion forums. Computers in Human Behavior, 28, 500-506.

Berger, R. (2015). Now I see it, now I don't: researcher's position and reflexivity in qualitative research. Qualitative Research, 15(2), 219-234. https://doi.org/10.1177/1468794112468475

Berthold, H. C., Hakala, C. M., \& Goff, D. (2003). An argument for a laboratory in introductory psychology. Teaching of Psychology, 30, 55-58. 
Boysen, G. A., Sawhney, M., Naufel, K. Z., Wood, S., Flora, K., Hill, J. C., \& Scisco, J. L. (2020). Mentorship of undergraduate research experiences: Best practices, learning goals, and an assessment rubric. Scholarship of Teaching and Learning in Psychology, 6(3), 212-224. https://doi.org/10.1037/st10000219

Branney, P., Reid, K., Frost, N., Coan, S., Mathieson, A. \& Woolhouse, M. (2019). A context-consent meta-framework for designing open (qualitative) data studies. Qualitative Research in Psychology, 16(3), pp. 483-502. doi: $10.1080 / 14780887.2019 .1605477$

Brewer, G., Dewhurst, A. M., \& Doran, D. (2012). Undergraduate research projects: Practice and perceptions. Psychology Learning \& Teaching, 11(2), 208-217. https://doi.org/10.2304/plat.2012.11.2.208

British Psychological Society (2017). Ethics Guidelines for Internet-mediated_Research. Retrieved from https://www.bps.org.uk/sites/www.bps.org.uk/files/Policy/Policy\%20\%20Files/Ethics\%20Guidelines\%20for\%20Internetmediated\%20Research\%20\%282017\%29.pdf

British Psychological Society (2019). Standards for the accreditation of undergraduate, conversion and integrated Masters programmes in psychology Retrieved from https://www.bps.org.uk/sites/bps.org.uk/files/Accreditation/Undergraduate\%20Accre ditation\%20Handbook\%202019.pdf

Buchanan, E. (2017). Considering the ethics of big data research: A case of Twitter and ISIS/ISIL. PloS one, 12(12), e0187155.

Button, K. S., Chambers, C. D., Lawrence, N., \& Munafò, M. R. (2020). Grassroots training for reproducible science: A consortium-based approach to the eEmpirical 
dbissertation. Psychology Learning \& Teaching, 19(1), 77-90.

https://doi.org/10.1177/1475725719857659

Button, K. S., Adams, R. C., Chambers, C. D., Lawrence, N., Pennington, C. R., Porter, L., ... Roy, J. (2020, April 30). Protocol: GW4 Undergraduate Psychology Consortium 2019/20. Retrieved from osf.io/p8qrv

Clark, V. L. P. (2019). Meaningful integration within mixed methods studies: Identifying why, what, when, and how. Contemporary Educational Psychology, 57, 106-111. https://doi.org/10.1016/j.cedpsych.2019.01.007

Coyle, D.K.T., Howard, S., Bibbey, A., Gallagher, S., Whittaker, A.C., Creaven, A-M. (2020). Personality, cardiovascular, and cortisol reactions to acute psychological stress in the Midlife in the United States (MIDUS) study. International Journal of Psychophysiology, 148, 67-74. https://doi.org/10.1016/j.ijpsycho.2019.11.014

Dautel, J.B. (2020). Applying a Collective Academic Supervision Model to the undergraduate dissertation. Psychology Teaching Review, 26(1), 18-26.

Detweiler-Bedell, B., \& Detweiler-Bedell, J. B. (2019). Undergraduate research teams that build bridges, produce publishable research, and strengthen grant proposals. Frontiers in Psychology, 10, 133. https://doi.org/10.3389/fpsyg.2019.00133

Erzberger, C., \& Prein, G. (1997). Triangulation: Validity and empirically-based hypothesis construction. Quality and Quantity, 31(2), 141-154.

Fanelli D (2010) "Positive" results increase down the hierarchy of the sciences. PLoS ONE, 5(4): e10068. https://doi.org/10.1371/journal.pone.0010068

Finn, C., \& Boland, P. (2021). Male family carers' experiences of formal support - a metaethnography. Scandinavian Journal of Caring Sciences. doi: 10.1111/scs.12919. 
Granger, J., Branney, P., Sullivan, P., \& McDermott, S. (2021, August 26). Ethical considerations in post-GDPR social media based research. https://doi.org/10.31234/osf.io/6fsw7

Habenicht, A.E., Gallagher, S., O’Keeffe, M-C., \& Creaven, A-M. (2021). Making the leap and finding your feet: A qualitative study of disclosure and social support at university for students with Type 1 diabetes. Journal of Health Psychology, 26(2):260-269. doi: $10.1177 / 1359105318810875$.

Healey, M., Lannin, L., Stibbe, A., \& Derounian, J. (2013). Developing and enhancing undergraduate final-year projects and dissertations. A National Teaching Fellowship Scheme project publication. Retrieved from: http://sure-network.ie/wpcontent/uploads/2015/08/3.-Developing-and-enhancing-undergraduate-final-yearprojects-and-dissertations-Chapter-8.pdf

Jahan, N., Naveed, S., Zeshan, M., \& Tahir, M. A. (2016). How to conduct a systematic review: A narrative literature review. Cureus, 8(11), e864. https://doi.org/10.7759/cureus.864

Janz, N. (2016). Bringing the gold standard into the classroom: Replication in university teaching. International Studies Perspectives, 17(4): 392-407. https://doi.org/10.1111/insp.12104

John, L. K., Lowenstein, G., \& Prelac, D. (2012) Measuring the prevalence of questionable research practices with incentives for truth telling. Psychological Science, 23 (5), $524-532$.

Johnson, R. B., Onwuegbuzie, A. J., \& Turner, L. A. (2007). Toward a definition of mixed methods research. Journal of Mixed Methods Research, 1(2), 112-133. https://doi.org/10.1177\%2F1558689806298224 
Kearney, M.W. (2019). rtweet: Collecting and analyzing Twitter data. Journal of Open Source Software, 4(42), 1829, https://doi.org/10.21105/joss.

Kitchin, R. (2013). Four critiques of open data initiatives. Retrieved from https://blogs.lse.ac.uk/impactofsocialsciences/2013/11/27/four-critiques-of-opendata-initiatives/\#author

Knight, R-A., \& Botting. N. (2016). Organising undergraduate research projects: Student-led and academic-led models. Journal of Applied Research in Higher Education, 8(4), 455-468.

Krishna, A., \& Peter, S.M. (2018) Questionable research practices in student final theses Prevalence, attitudes, and the role of the supervisor's perceived attitudes. PLOS ONE, 13(8): e0203470. https://doi.org/10.1371/journal.pone.0203470

Lee, R.S., Hermens, D.F., Porter, M.A., \& Redoblado-Hodge, M.A. (2012). A meta-analysis of cognitive deficits in first-episode major depressive disorder. Journal of Affective Disorders, 140(2), 113-124. doi:10.1016/j.jad.2011.10.023

Linn, M.E., Palmer, E., Barranger, A., Gerrard, E., \& Stone, E. (2015). Undergraduate research experiences: Impacts and opportunities. Science, 347, 6222. DOI: $10.1126 /$ science. 1261757

Madan, C. R., \& Teitge, B. D. (2013). The benefits of undergraduate research: The student's perspective. The Mentor: An Academic Advising Journal. Retrieved from http://dus.psu.edu/mentor/.

Moshontz, H., Campbell, L., Ebersole, C. R., IJzerman, H., Urry, H. L., Forscher, P. S., ... Chartier, C. R. (2018). The Psychological Science Accelerator: Advancing Psychology through a Distributed Collaborative Network. Advances in Methods and Practices in Psychological Science, 1(4), 501-515. https://doi.org/10.1177/251524591879760 
National Association of Colleges and Employers. (2019). Job Outlook 2019. Report retrieved from https://www.odu.edu/content/dam/odu/offices/cmc/docs/nace/2019nace-job-outlook-survey.pdf on 14th October 2020.

Norcross, J. C., Hailstorks, R., Aiken, L. S., Pfund, R. A., Stamm, K. E., \& Christidis, P. (2016). Undergraduate study in psychology: Curriculum and assessment. The American Psychologist, 71(2), 89-101. https://doi.org/10.1037/a0040095

Nordentoft, H. M., Thomsen, R., \& Wichmann-Hansen, G. (2013). Collective Academic Supervision: A model for participation and learning in higher education. Higher Education, 65(5), 581-593.

Nosek, B. A., Beck, E. D., Campbell, L., Flake, J. K., Hardwicke, T. E., Mellor, D. T., ... \& Vazire, S. (2019). Preregistration is hard, and worthwhile. Trends in Cognitive Sciences, 23(10), 815-818.

Palmer, C., \& Bartlett, J. E. (2021, June 6). "To have been forgiven by God and those I have wronged first": A mixed methods study on self-forgiveness. https://doi.org/10.31234/osf.io/eca5j

Perlman, B., \& McCann, L. I. (2005). Undergraduate research experiences in psychology: A national study of courses and curricula. Teaching of Psychology, 32(1), 5-14. https://doi.org/10.1207/s15328023top3201_2

Pownall, M. (2020). Pre-registration in the undergraduate dissertation: A critical discussion. Psychology Teaching Review, 26(1), 71-76.

Psychological Society of Ireland Undergraduate Accreditation Guidelines (2019). Retrieved from https://www.psychologicalsociety.ie/accreditation/PSI$\underline{\text { Accredited-Undergraduate-Courses-3. }}$. 
PsyTeachR (n.d). PsyTeachR. Retrieved on April 26 2021 from https://psyteachr.github.io/

Roberts, L.D., \& Seaman, K. (2018). Students' experiences of undergraduate dissertation supervision. Frontiers in Psychology, https://doi.org/10.3389/feduc.2018.00109.

Rocher, L., Hendrickx, J.M. \& de Montjoye, YA. Estimating the success of reidentifications in incomplete datasets using generative models. Nature Communications, 10, 3069 (2019). https://doi.org/10.1038/s41467-019$\underline{10933-3}$

Ruggiano, N., \& Perry, T. E. (2019). Conducting secondary analysis of qualitative data: Should we, can we, and how? Qualitative Social Work, 18(1), 8197. https://doi.org/10.1177/1473325017700701

Shultz, K.S.; Hoffman, C. C.; \& Reiter-Palmon, R. (2005).Using Archival Data for I-O Research: Advantages, Pitfalls, Sources, and Examples. Psychology Faculty Publications. 5. https://digitalcommons.unomaha.edu/psychfacpub/5

Szollosi, A., Kellen, D., Navarro, D.J., Shiffrin, R., van Rooij, I.,Van Zandt, .T., \& Donkin, C. "Is preregistration worthwhile." Trends in Cognitive Sciences 24, no. 2 (2019): 94-95.

Sugiura, L., Wiles, R., \& Pope, C. (2017). Ethical challenges in online research: Public/private perceptions. Research Ethics, 13(3-4), 184-199. doi:10.1177/1747016116650720

Tate, J. A., \& Happ, M. B. (2018). Qualitative Secondary Analysis: A Case Exemplar. Journal of Pediatric Health Care, 32(3), 308-312. https://doi.org/10.1016/j.pedhc.2017.09.007 
van't Veer, A. E., \& Giner-Sorolla, R. (2016). Pre-registration in social psychology-A discussion and suggested template. Journal of Experimental Social Psychology, 67, 212.

Weston, S. J., Mellor, D. T., Bakker, M., Van den Akker, O., Campbell, L., Ritchie, S. J., ... DeHaven, A. C. (2021, August 9). Secondary Data Preregistration. Retrieved from osf.io/x4gzt

Wilkinson, M., Dumontier, M., Aalbersberg, I. et al. The FAIR Guiding Principles for scientific data management and stewardship. Sci Data 3, 160018 (2016). https://doi.org/10.1038/sdata.2016.18

Wills, A.J. (n.d). Teaching research methods in R. Research Methods in R. Retrieved on $15^{\text {th }}$ March 2021 from https://ajwills72.github.io/rminr/rminrinpsy.html

Williams, M.L., Burnap, P., Sloan, L. (2017). Towards an ethical framework for publishing Twitter data in social research: Taking into account users' views, online context and algorithmic estimation. Sociology, 51(6), 1149-1168. doi:10.1177/0038038517708140 